\title{
Study of Event Topology for a new Fast Primary Vertex Finder for the ATLAS Trigger
}

\author{
Robert Langenberg, on behalf of the ATLAS Collaboration
}

\begin{abstract}
This document presents a transform-based approach to primary signal vertex finding feasibility analysis. An algorithm to find primary vertices has been developed using only Inner Detector measurements. Based on the information available to the algorithm, a metric has been developed to identify the signal vertex. The feasibility analysis demonstrates the theoretical distinguishability of different signal events and pileup with this metric. The results show that the majority of event types are easily distinguished. The algorithm can be used in the high level trigger. At this stage of computation, event types can be identified with the trigger information, such that this algorithm can be chosen only for appropriate events. An implementation of the algorithm is used to run simulations with increasingly realistic scenarios. This allows the quantification of the algorithm performance and to attribute inefficiencies to specific effects. In the most realistic scenario, the distinction rate reduces only by a small margin, remaining higher than $90 \%$ for events with good results in the feasibility analysis, also for high pileup scenarios. Countermeasures are presented for the effects reducing efficiency.
\end{abstract}

\section{INTRODUCTION}

$T$ RACKING is one of the computationally most expensive tasks of event reconstruction. ATLAS tracking algorithms in place in the offline reconstruction and in the trigger have high complexities, leading to very long runtimes for higher luminosities and higher pileup in particular. Pileup denotes collisions simultaneous to the signal that do not contain interesting physics and therefore only contribute noise. The presented algorithm is based on a solution that was in place for the ATLAS Trigger during LHC's Run 1. It has a linear complexity, meaning that the runtime increases at the same rate as the size of the problem, which here is the number of particles in the detector. The problem with this solution is that it only works well for low levels of pileup as it is severely limited by high background levels. This background is the result of arbitrary combinations of information from different particles falsely identified as tracks, so called fake tracks. The number of fake tracks increases with pileup.

While this algorithm cannot be used in its present form, a study presented in this paper has been conducted showing the theoretical applicability of the approach under ideal conditions and in the absence of noise. Subsequently an extension to the algorithm with various improvements including elimination of

Manuscript received December 7, 2015. This work was supported in part by Innsbruck University and Technische Universität München.

R. Langenberg is with Innsbruck University, Austria

(e-mail: robert.langenberg@cern.ch). the noise will be presented. A proof of concept implementation with all improvements is presented in this paper, which can be used for further efficiency studies and can easily be included in the current ATLAS software framework.

\section{Event Topology STUdy}

Part of the work presented here is a study showing applicability of the approach in case of perfect information in order to distinguish pileup vertices from a signal vertex. In the ideal case the information necessary could be collected using various layers of silicon detectors on each of which an ideal particle would ionize the silicon at the point it passed through. For the ATLAS Inner Detector (ID), the ionization is read out and converted to spatial information called a space point. The ID silicon detector is a tracker consisting of both Pixel Tracker and Semi Conductor Tracker (SCT). Different event types have been chosen covering different scenarios of event signatures in the ID. This study establishes an upper limit for the maximum efficiency of the vertex finder algorithm with the tested metric. A particle's transverse momentum $\left(\mathrm{p}_{\mathrm{T}}\right)$ is chosen as distinguishing property because particles from the signal usually have high $\mathrm{p}_{\mathrm{T}}$ and in most cases other particles resulting from the interaction will also have a $\mathrm{p}_{\mathrm{T}}$ above average.

The study distinguishes between low $\mathrm{p}_{\mathrm{T}}$ pileup, which makes up the majority of all interactions, and high $\mathrm{p}_{\mathrm{T}}$ pileup, which is only a small fraction of all interactions. The study is based on Monte Carlo simulations and their pileup distribution. Various metrics have been considered combining number of tracks and their respective transverse momenta.

In order to simulate pileup effects, ATLAS uses presimulated proton-proton minimum bias interactions. Most pileup vertices are easily distinguished from signal events and in fact often not even detected, as they may have few or no charged particles in the acceptance range of the tracking detector. However, some of the minimum bias events have particles with $\mathrm{p}_{\mathrm{T}}$ similar to that of signal events. With increasing pileup, the probability for one of the pileup vertices to have more high $\mathrm{p}_{\mathrm{T}}$ charged particles than the signal vertex increases. Therefore, the probability to correctly identify a signal vertex decreases with higher pileup.

\section{TRUTH ANALYSIS}

To establish an upper limit, the truth information is used, referring to the generated particle information, which is the simulation input rather than the interpreted simulation output. The goal is to find a metric that - using this information - 
successfully identifies different signal events from high levels of pileup. Four different event types have been chosen to cover strongly differing ID signatures.

- The muons from a $Z \rightarrow \mu^{+} \mu^{-}$decay leave two highenergy tracks in the silicon detectors with low probability for significant energy loss or decay. Probability is high that transverse momentum is in the region of multiple $\mathrm{GeV}$. The name used for this process in figures and tables is Zmumu.

- A $t \bar{t}$ process has various decay modes. In this study we look at the full hadronic (henceforth: allhad) decay mode, and the case where at least one of the $\mathrm{W}$ bosons decays leptonically (nonallhad).

- A signal event selected to show the limitations of the algorithm is the Higgs boson decay to two photons. The two photons carrying the whole energy of the Higgs do not leave a direct trace in the ID. The signal therefore does not contribute to finding the primary vertex.

Besides the particles from the signal, there are always arbitrary other particles from this interaction, on top of the discussed signal signatures. Various metrics have been tried combining track $\mathrm{p}_{\mathrm{T}}$ and number of charged particle tracks per vertex, as these can be measured easily within the original vertexing algorithm. Considerations for the metric were favoring high $\mathrm{p}_{\mathrm{T}}$ and number of high $\mathrm{p}_{\mathrm{T}}$ tracks. Because multiple pileup collisions may occur close to each other, which would subsequently be reconstructed as a single vertex, a high number of low $\mathrm{p}_{\mathrm{T}}$ tracks should not increase the likelihood to identify a vertex as the signal vertex. The best distinction rate was achieved with $\Sigma\left(\frac{\mathrm{p}_{\mathrm{T}} \mathrm{p}_{\mathrm{T}}}{\# \text { tracks }}\right)$, with \#tracks denoting the number of tracks found for a vertex candidate. The rating of the different event types in Fig. 1 shows that most signal events, with the exception of a Higgs to two photons decay, can be very likely distinguished from minimum bias events using this metric.

The probability for the signal vertex being among the three highest rated vertices is given for four different signal event types in TABLE 1. Four different pileup scenarios with 20, 40, 80 and 160 simultaneous interactions in one bunch crossing are evaluated. Requiring the signal vertex to be among the top three rated vertices requires reconstructing all three vertices to achieve the quoted efficiencies. Since reconstruction with knowledge of the vertex location is orders of magnitude faster than without using the location, this still allows for efficient reconstruction.

TABLE 1: TRUth ANALYSIS For CHARGED PARTICLES WITH $\mathbf{P}_{\mathbf{T}}>1 \mathrm{GEV}$. PROBABILITY TO SUCCESSFULLY DISTINGUISH THE DIFFERENT SIGNAL VERTICES FROM PILEUP INTERACTIONS WITH THE GIVEN METRIC.

\begin{tabular}{lllll} 
Pileup & $t \bar{t}$ allhad & $t \bar{t}$ nonallhad & Zmumu & $\mathrm{H} \gamma \gamma$ \\
\hline 20 & $100 \%$ & $100 \%$ & $99.9 \%$ & $75.7 \%$ \\
40 & $100 \%$ & $100 \%$ & $99.7 \%$ & $63.6 \%$ \\
80 & $99.9 \%$ & $99.9 \%$ & $99.6 \%$ & $52.8 \%$ \\
160 & $99.9 \%$ & $99.7 \%$ & $99.1 \%$ & $43.7 \%$ \\
\hline
\end{tabular}

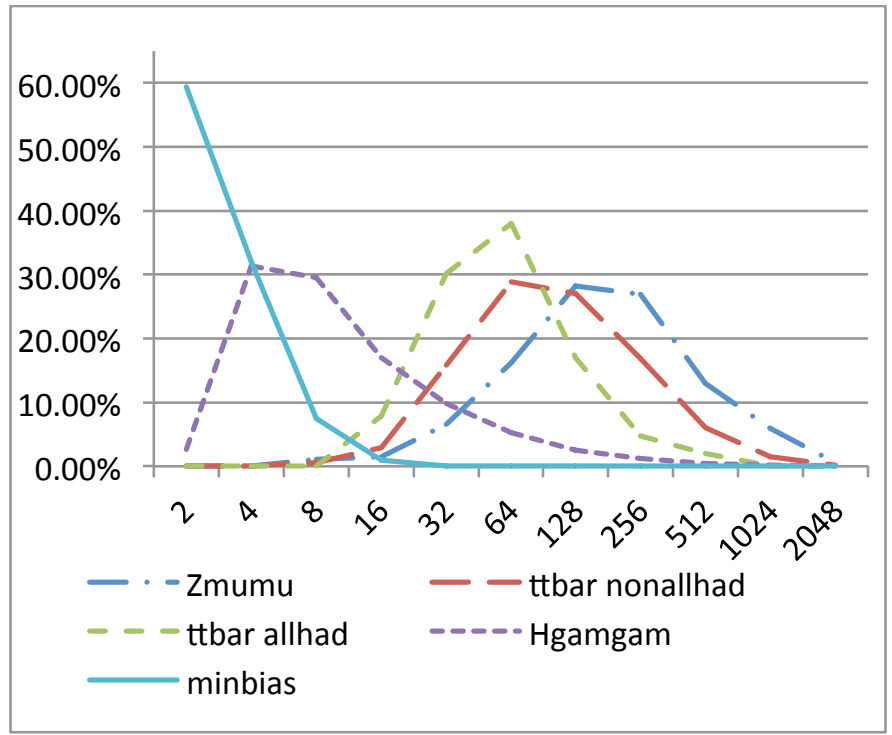

Fig. 1: Metric rating of different event types. The $\mathrm{x}$-axis denotes the rating according to the metric and the $y$-axis denotes the percentage of entries in this bin. With the exception of the Higgs to two photons decay, all signals event distributions only overlap in the tails with the minimum bias distribution.

As the results show, this algorithm is not efficient for all types of events. Some signals, such as the two photons from a Higgs decay do not leave a trace in the silicon detector. Trigger information during high-level trigger reconstruction is already available; hence it is possible to apply the algorithm only to events for which a sufficient number of charged particle tracks in the Inner Detector can be expected from the signal. For other tested event types, these figures are sufficiently high, especially taking into account that typically only regions of interest are reconstructed and not the whole event. It should be noted that for this analysis, it has not been tested if the high complexity algorithms currently in place would find the signal or if ATLAS would have detected it.

\section{Algorithmic Details of the Vertex Finder}

The study suggests that under ideal conditions it is possible to distinguish event topologies from one another using only ID space points. The existing algorithm uses combinations of Pixel Tracker space points to calculate the particle energy and origin. While this is enough to find all tracks originating from the signal, it will also generate many false positives. The algorithm has been implemented from scratch to account for changes in the geometry for Run 2 implementation of noise elimination techniques and other improvements in physics performance and inclusion of the silicon detector endcaps.

\section{A. Principle of the algorithm}

The algorithm uses combinations of two pixel detector space points to determine the origin of a possible track. It uses an extrapolation in the r-z plane. It exploits that particles traversing a homogeneous magnetic field, which bends them in the azimuthal direction $\varphi$, are on a straight line in r-z. To avoid incompatible combinations, the space points are sorted in $\varphi$-bins such that only combinations above a minimum 
energy are considered. Such higher energy particles have an increased likelihood of forming a helical track without distortions and contribute to finding the primary vertex.

As mentioned, a major problem for the application of this algorithm is the noise, as many space point combinations with feasible $\varphi$ differences do not belong to a track. To effectively check if a combination belongs to a track, the existence of additional space points on the calculated helical trajectory can be tested. To minimize the CPU cost for these tests, all space points from silicon layers, the SCT and the Pixel Tracker, are binned in a 3D histogram by $\varphi$ and $\mathrm{z}$ for barrel space points and in $\varphi$-r for space points on the endcaps. Bin sizes must be adjusted for a tradeoff between noise elimination and allowing for deviations from the ideal assumed conditions. Because the subsequent layer can be calculated using the trajectory, the radius for space points in the barrel respectively the $\mathrm{Z}$ coordinate in the endcap is known. With the next layer known and the angle and curvature of the track being calculated from the two initial pixel space points, it is possible to accurately determine the coordinates on the next detector surface, ref. Fig. 2.

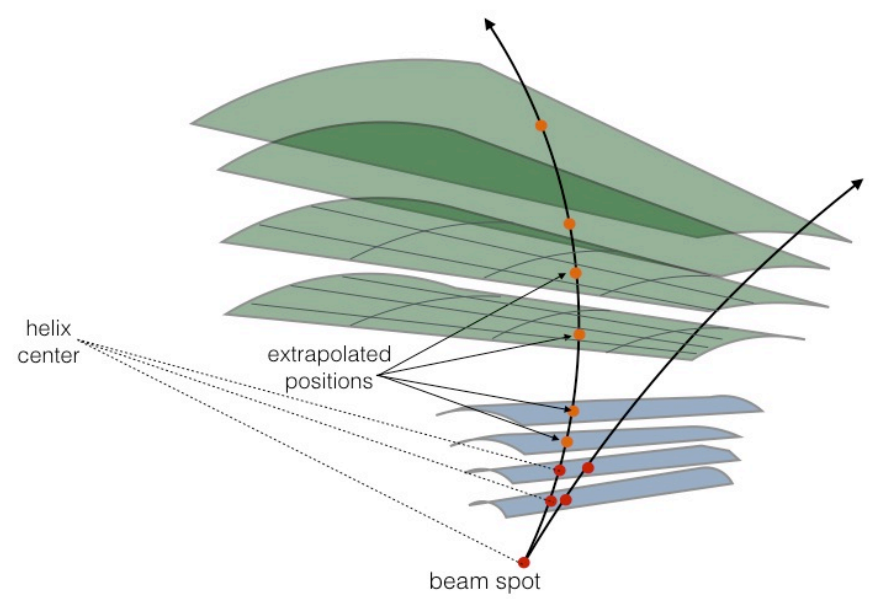

Fig. 2. Schematic of how layers are binned in $\mathrm{z}$ and $\boldsymbol{\varphi}$. The path of a potential track is calculated using two space points and the beam spot. If sufficient space points are found, a track pointing to the beam spot is considered found.

This way, a check for the existence of additional compatible space points completely avoids scanning through a list of space points to find a match but just requires a lookup in the histogram instead. By setting a single bit corresponding to the layer in the histogram for each space point, the check becomes the inexpensive calculation of a straight line through two points in combination with a single bit test. By requiring at least six compatible space points, the probability for noise forming a helical track quickly drops to almost zero. The ATLAS Run 2 geometry is designed to generate at least eight space points for a track within the covered phase space, so requiring six space points still allows for some inefficiency. Each time sufficient compatible entries for a pair of two pixel space points are found, the location with respect to the beam axis is stored in a vertex histogram together with the energy of this track. After looping over all feasible space point combinations, the individual track energies and number of tracks for each vertex bin are used to calculate the metric to score each bin. The three highest rated bins are returned and assumed to contain the primary vertex of the signal interaction.

\section{B. Further improvements to the algorithm}

Prediction of the potential space point location on a subsequent layer from pixel space point combinations is limited by the resolution accuracy of these layers and the distance between these layers. Slight inaccuracies can lead to missing the corresponding SCT histogram bin by one, especially for border cases where the SCT space point would lie on the border of a bin. To account for these inaccuracies, when inserting a hit into the histogram the eight surrounding bins are filled as well. This improves finding compatible bins without significantly adding noise. Noise levels are low for even high pileup scenarios because of the high resolution of the $\mathrm{z}-\varphi$ respectively $\mathrm{r}-\varphi$ histograms. These histograms have in the order of $10^{6}$ bins but only about $10^{3}$ to $10^{4}$ space points per detector layer.

Another inaccuracy related to finding the compatible SCT space points is that the barrel is not a perfect cylinder but consists of flat overlapping modules arranged around the center. Similarly, the endcaps have overlapping modules to avoid cracks where particles could pass undetected. This leads to differences in $\mathrm{r}$ of up to $4 \mathrm{~cm}$ between space points on the innermost respectively on the outermost position of a module for the barrel and similar differences for the overlapping endcap modules. A lookup in the 3D histogram based on an $r$ respectively $\mathrm{z}$ value with a $2 \mathrm{~cm}$ error can lead to a wrong lookup. This is improved by shifting each space point towards the average radius of the barrel respectively average $\mathrm{z}$ value in the endcaps in the direction of the detector center. Although this is not accurate as tracks can origin from the whole interaction zone, which is around $\pm 15 \mathrm{~cm}$ from the detector center. The correction direction deviates from the actual track direction most in the central barrel regions, as the distance a particle travels is shortest in this case. The measure is nonetheless helpful as the correction in $\mathrm{z}$ is minimal in barrel areas close to the ATLAS center. The $\varphi$ direction cannot be corrected in the same way, as it depends on a particle's charge and transverse momentum.

\section{IMPLEMENTATION AND TESTING WiTH IDEALIZED DATA}

The vertex finder algorithm has been implemented with the aim of running tests on data in order to compare the distinguishability of vertices using the algorithm. The idealized data initially used for a test contains no energy loss and a homogeneous magnetic field. It was produced with the fast ATLAS track simulation program FATRAS [7]. The vertices are always at the center of the detector and each track is tested individually to eliminate the effect of noise. The detector geometry for this test is the same that is used for both reconstruction and simulation. The ideal homogeneous field and the missing energy loss lead to charged particles following perfectly helical paths. This leaves only two reasons why a track cannot be found: inaccuracies in the calculation and the 
detector geometry. Numerical inaccuracies have shown to be insignificant. The geometry influences the result of the algorithm in different ways. First, it deviates from the idealized geometry with flat endcap regions and perfectly cylindrical barrel regions assumed in the algorithm. Second, only particles with pseudorapidity $|\eta| \leq 2.5$ can generate enough space points to be detected. Therefore, a particle is considered findable if its pseudorapidity $|\eta| \leq 2.5$ and it generated at least six space points. Instrumenting the simulation shows that about $45 \%$ of all charged particles with $\mathrm{p}_{\mathrm{T}}>1 \mathrm{GeV}$ are unfindable. Results from running the algorithm under these conditions are shown in TABLE 2.

TABLE 2: ANALYSIS WITH IDEALIZED PARTICLE TRACKS AND REALISTIC DETECTOR FOR CHARGED PARTICLES WITH $\mathbf{P}_{\mathrm{T}}>1$ GEV. PROBABILITY TO SUCCESSFULLY DISTINGUISH THE DIFFERENT SIGNAL VERTICES FROM PILEUP INTERACTIONS WITH THE GIVEN METRIC.

\begin{tabular}{lllll} 
Pileup & $t \bar{t}$ allhad & $t \bar{t}$ nonallhad & Zmumu & $\mathrm{H} \gamma \gamma$ \\
\hline 20 & $99.8 \%$ & $99.7 \%$ & $99.2 \%$ & $61.1 \%$ \\
40 & $99.6 \%$ & $99.2 \%$ & $98.7 \%$ & $48.6 \%$ \\
80 & $98.9 \%$ & $97.9 \%$ & $96.6 \%$ & $38.1 \%$ \\
160 & $98.3 \%$ & $94.8 \%$ & $93.1 \%$ & $30.1 \%$ \\
\hline
\end{tabular}

As the results show, the geometry influences the efficiency for different signals to a different extent, even though $99 \%$ of all tracks with sufficient space points are found for all event types. Missing one percent of the reconstructible tracks is not seen as a major problem, especially taking into account that a far bigger part is lost due to the detector geometry. This high number of missed tracks also explains the reduced performance for higher pileup scenarios compared to the truth study, because it becomes more likely that any high $\mathrm{p}_{\mathrm{T}}$ pileup vertex loses less information than the signal. For the event types for which a good theoretical distinguishability has been shown using the metric on the truth information, the probability to identify the signal vertex is still reasonably high. To reduce the effect of losing $1 \%$ of findable tracks, corrections to pixel space points similar to the ones applied in the SCT can be applied. To avoid these corrections from having negative effects for vertices far from the point to which they are shifted (the ATLAS center for the SCT), the correction can be applied multiple times corresponding to $\mathrm{Z}$ slices in the interaction region. This would not increase the number of space point combinations, but would require refilling the histogram for pixels a number of times equal to the number of slices. The number of slices can be calculated such that the number of times the histogram has to be refilled is minimal, with the error in $\mathrm{z}$ still being smaller than the z-binning.

A second test was performed with a more realistic setting, though without including any effects of background. The first scenario has been changed to include energy loss through ionization and multiple scattering in the detector and a realistic magnetic field. Energy loss leads to a reduced helix radius and therefore a change in $\varphi$. The direction of change in $\varphi$ is known (energy can only decrease), so larger $\varphi$ binning can reduce the effect or testing $\varphi$-bins for smaller helix radii. This would increase the number of fake tracks found. If required, a tradeoff between efficiency and noise can be made in separate tests. Multiple scattering leads to additional randomization of the particle path, which can be described by a smearing of the assigned helical trajectory. The realistic magnetic field has a slight change in direction towards the endcaps and a slightly different strength when comparing center and at larger radii. While these could in part be incorporated in the algorithm to calculate the bin of the next space points, it would increase the complexity and computational cost of this otherwise very fast and simple algorithm. Results in TABLE 3 suggest that the expectable maximum improvement would be small when comparing it to TABLE 2, especially taking into consideration that effects from multiple scattering remain. Particles affected by energy loss can only be partially reconstructed with expensive methods such as the Kalman filter used in offline reconstruction. As in the previous test, simulated collisions only take place the ATLAS center, which is not expected to significantly influence the results.

TABLE 3: ANALYSIS WITH REALISTIC PARTICLE TRACKS AND REALISTIC DETECTOR FOR CHARGED PARTICLES WITH $\mathbf{P}_{\mathbf{T}}>1$ GEV. PROBABILITY TO SUCCESSFULLY DISTINGUISH THE DIFFERENT SIGNAL VERTICES FROM PILEUP INTERACTIONS WITH THE GIVEN METRIC.

\begin{tabular}{lllll} 
Pileup & $t \bar{t}$ allhad & $t \bar{t}$ nonallhad & Zmumu & $\mathrm{H} \gamma \gamma$ \\
\hline 20 & $99.7 \%$ & $99.5 \%$ & $98.9 \%$ & $56.1 \%$ \\
40 & $99.3 \%$ & $98.6 \%$ & $98.0 \%$ & $43.2 \%$ \\
80 & $98.7 \%$ & $96.1 \%$ & $96.0 \%$ & $32.8 \%$ \\
160 & $97.7 \%$ & $91.75 \%$ & $92.8 \%$ & $25.5 \%$ \\
\hline
\end{tabular}

While in this test only $95 \%$ of the tracks are found for any type of event, the distinction rate is still comparable to the one with idealized tracks, though dropping notably for high pileup scenarios. Although the performance could be slightly improved with the discussed methods, it is arguable if it would justify the increased computational costs.

\section{CONCLUSION AND OUTLOOK}

This conference record describes the physics performance of an improved vertex finding algorithm when applied to different types of data. A metric to distinguish the two using data available in the ATLAS Inner Detector has been developed. A truth analysis on simulated data establishes an upper boundary for the distinction rate of pileup interactions from signal with the chosen metric. The results are compared to simulated events with an implementation of the algorithm. A simple metric working on individual tracks is used. The implementation shows that the algorithm gets results close to the upper boundary for low pileup scenarios with gracefully decreasing performance with higher pileup scenarios. The algorithm additionally implements improvements to the original algorithm using simple and fast operations. The analysis will be expanded to measure computing performance of this approach and parallelizability. A selection to eliminate events that would not trigger was not performed, but it is expected that results would have been closer to $100 \%$ distinction if events with too many lost tracks had been filtered out beforehand. A preselection has only taken place for the $Z \rightarrow \mu^{+} \mu^{-}$decay signal. If at least one muon was findable, the event was accepted. Consequently, further studies are necessary to establish the full applicability of this algorithm to the ATLAS trigger system. 


\section{REFERENCES}

[1] H. Drevermann, D. Kuhn, P. Luthaus and B. S. Nilsson, A linear algorithm to filter and group 3D hits of the discrete tracking layers, ATL-SOFT-97-035 ; ATO-PN-35

[2] P. Bernat, Architecture and performance of the Inner Detector trigger of the ATLAS detector, Proc. of CHEP 2012

[3] ATLAS Collaboration, The ATLAS experiment at the CERN Large Hadron Collider, JINST 3 (2008) S08003

[4] S. Armstrong, Algorithms for the ATLAS high-level trigger, DOI: 10.1109/TNS.2004.828620

[5] I. Christidi, Performance of the ATLAS Inner Detector trigger algorithms in pp collisions at $\sqrt{s}=900 \mathrm{GeV}$, RT 2010, IEEE Trans. Nucl. Sci. 58 , 4, Part 1 (2011)

[6] N. Konstantidinis and H. Drevermann, Fast tracking in hadron collider experiments, ACAT 2000 ISBN: 0-7354-0023-7

[7] A. Salzburger, A. Wildauer, S. Fleischmann and T. Lenz, The new fast ATLAS track simulation engine (Fatras), Proc. of CHEP 2006 